\title{
Body Specificity Hpothesis: Different Bodies Develop Correspondingly Different Brains and Minds
}

\author{
Quan Tang ${ }^{1}$, Tingjun Li ${ }^{2}$, Junyan Fang ${ }^{3, *}$ \\ ${ }^{1}$ Faculty of Psychology, Beijing Normal University, Beijing, China \\ ${ }^{2}$ Annie Wright Schools, Seattle, WA, United States \\ ${ }^{3}$ Faculty of Business Foreign Languages, Shanxi University of Finance and Economics, Shanxi, China, \\ junyan_fang@163.com
}

\begin{abstract}
This electronic document is a "live" template. In the current research of cognitive psychology, the field of embodied cognition has become an important part of cognitive science. The introduction of the body-specific hypothesis adds important pieces to the puzzle of the theoretical structure of embodied cognition. The body-specific hypothesis (Casasanto, 2009) suggests that the content of our mind depends to a great extent on our body structure. In this article, we reviewed studies for neurological and brain imaging and behavioral evidence to support the body-specific hypothesis based on the hands and other body parts including foot laterality, general fluency and pain perception. In terms of handedness, we mainly focused on the perspective of action verbs, action and emotion valence, approach motivation and motor control, visuospatial attention and metaphor explanation. In terms of some studies, though did not mention the body specific theory explicitly, they provided supportive results towards the theory. For evidence of other parts of the body, we try to find the relationship between metaphors and other body experiences other than handedness, so as to expand the boundaries of the body specificity hypothesis. While providing evidence for the body specificity hypothesis (BSH), we also try to prove that the body experience influences human cognition through the above content, that is, cognition is embodied. Additionally, we also offered possible research directions for the further exploration and application of the hypothesis, including cross-cultural examination, motivational or emotional factors, and the effect of neuro abnormality.
\end{abstract}

Keywords- body-specificity hypothesis (BSH), concepts, embodied cognition, handedness

\section{INTRODUCTION}

Cognitive psychology has been the mainstream of western psychology since the 1960s. At that time, computer model of mind [5] was dominant in cognitive psychology, which described human thinking as computerized information processing. Researchers of this period believed that human thinking is a kind of process which only manipulate symbol without understanding the meaning, just like a computer [35]. If the brain is compared to the "hardware" of the computer, then cognition is the "software" or "program" running on this "hardware" independent of the body, which is the view of disembodiment. Later, the connectionist model, which is based on the both neural web-based structure and the principle of parallel processing, came into the field of cognitive psychologists. It claims that the brain is a complex information processing system composed of an extremely large number of interconnected neurons, and thus constructs an artificial neural network, trying to find out how cognition emerges in the complex connections and parallel distributed processing. Compared with behaviorism, both of these views shift the focus of psychologists' attention to internal psychological processes and strive to explore the cognitive mechanisms that regulate behavior. However, influenced by the development of cognitive linguistics, cultural anthropology, philosophy, robotics, artificial intelligence and other subjects, cognitive science researchers reconsidered the question that how does thinking work, and then put forward embodied cognition, a brand-new research field of cognitive psychology.

The concept of embodiment originates from the debate between mind-body monism and mind-body dualism in philosophy. Dualism holds that the mind and body, including the brain, are independent of each other 
and that there is no overlap between their functions. Based on this theory, human cognition should be a completely independent system, and the processing of information should not be affected by the unexpected factors of the cognitive system itself. However, Descartes criticized it, arguing that since the mind is locked in the body, it occupies a special space and faces a specific direction at all times. These undeniable facts form part of the basis of embodied cognition [38]. The traditional view of cognitive science is that the concepts in our minds are stable across time and across individuals, but this view is not supported by empirical research. On the contrary, there are abundant evidence that human representations of concept vary from person to person and are subject to physical and experience change. The embodied cognition view holds that a cognitive process emerges from the physical attributes of the body, accounting for the content of cognition, for example the concept, by appeal to the nature of the body [41]. Theorists of embodied cognition claim that thinking and cognition are largely dependent on and originated from the body. The structure of the body, the neural structure, the activity mode of the sensory and motor systems determine how we perceive the world, determine our thinking style, and shape the way we see the world. On account of this standpoint, Casasanto (2009) [16] put forward the definition---the Body Specificity Hypothesis (BSH).

The BSH, which expounds the link between physical characteristics and mental content is a crucial part of embodied cognition, adding an important piece to the puzzle of cognitive psychology. When we use our minds, our bodies are also constantly exposed to the situation, and thus will continue to influence the formation of corresponding representations. Meanwhile, the BSH [16] suggests that the content of our mind depends to a great extent on our body structure. In other words, different bodies should lead to different thinking. What we can easily understand is that physical characteristics may influence the representation of objects and actions, for example, red and green color blindness may represent apples differently. However, abstract concepts seem different, such as righteousness and immorality as well as deception and genuineness. How do we represent concepts that are not simulations of sensorimotor experience? Mr. Casasanto and his colleagues [14-16] discuss the question in terms of valence and spatial orientation, as in English metaphors for good and bad. His works shows that the mapping from spatial location to affective valence was different between right- and left-handed individuals. Right-handers tend to associate right-handers with positive thoughts and left-handers with negative thoughts, while left-handers tend to associate right-handers with negative thoughts and lefthanded with positive thoughts. Because left-handers associated positive thoughts with the left side of the space, the effect of linguistic experience was eliminated.
Such experimental results indicate that the use of dominant hand has an impact on the construction of valence metaphors for spatial orientation. Moreover, it upholds that individuals possessing different physical characteristics ought to shape with different mental representations correspondingly.

The field of research at $\mathrm{BSH}$ has accumulated rich results to support this hypothesis. For example, some researchers have used neuroimaging and brain imaging methods to explore the differences between spatial orientation and association of valence (good vs. bad) representations in left-handed and right-handed people [40]. Some studies have used theta-burst repetitive transcranial magnetic stimulation (rTMS) to regulate neural activity and demonstrated through functional magnetic resonance imaging (fMRI) that the bodyspecific activation of the motor system plays a functional role in the language processing of hand movements [39]. In addition, a large number of behavioral experiments also support BSH [8, 9, 20, 42]. In these studies, participants with different dominant hands were asked to select stimuli in different spatial locations to reflect the different spatial location metaphors of body (dominant hand) characteristics for positive and negative abstract concepts. So far, the study of handedness has accumulated a tremendous amount and is abundant enough. Future research is likely to focus on other parts of the body, looking for more effects of body experiences on cognition, such as nodding the head (indicating agreement or approval) and shaking the head (indicating disagreement or disapproval). Apart from this, the participants with acquired disabilities and congenital disabilities will also be concerned with the aim of obtaining greater physical differences. As mentioned above, the simulation of red-green color blindness for the abstract concept of color metaphor is also worth discussing. Of course, cultural factors as one of the boundary conditions of BSH should also be considered. Metaphors of abstract concepts are not identical in different cultures, which leads to the value of cross-cultural research.

In this article, we review the results of previous empirical studies on the body-specificity hypothesis based on the hands. The good and right associations between left- and right-handers have been well discussed, but some researchers are increasingly focusing on other parts of the body. We will also summarize existing research on other body parts (such as feet, head, etc.) with the aim of providing a series of available evidence to support future research and inspire new areas of research. By summarizing previous studies, we point out new directions for the study of the body specificity hypothesis in this paper, with the hope of facilitating future developments in this field. The theory related to metaphor in embodied cognition will also be partially covered, but these are not the focus of this paper, therefore we will not do a detailed discussion. 


\section{DiSCUSSION ABOUT HANDEDNESS}

From previous studies, it can be figured easily out that researchers prefer to use handedness as their testbed to support the BSH and they have done plenty of exploration in this field. As an initial test bed upheld the $\mathrm{BSH}$, these original works concentrated on "how handedness, genetic or induced, influences thinking" [11]. Meanwhile, Casasanto in 2011 put forward primary definition of handedness is that "right- and left-handers tend to form systematically different mental images, create different word meanings, and arrive at opposite judgments about the same objects in the world" [11]. So, what factors are associated with handedness in different environments?

\subsection{Semantics: Action Verbs}

Embodied cognition theory suggests that language has an inseparable connection with bodily experience (cited Willems, Hagoort, \& Casasanto, 2009). From this body of knowledge, the meaning of an individual word accounted by Casasanto (2011), namely, is to 'build up an implicit mental simulation of its referent by the help of preceptive and actional regions in our brain'. [11] From previous studies, many researchers did many tests to support this point of view before 2008. However, their essays just give the vital information that when participants read an individual word such as kick, pick, and lick attached to action verbs, they tend to move the effector, in which refers to the activation of premotor cortex, most related to the verbs involving their other part of body (cited Casasanto, 2011). Until 2009, the findings from Willems et al. told us that action verbs did influences handedness. The results of data show that when making lexical decisions for hand-operated verbs, right-handed individuals preferentially activate the left anterior motor area (as compared with the non-handoperated verbs), while left-handed persons preferentially activate the right anterior motor area (cited Willems, Hagoort, \& Casasanto, 2009). More importantly, they further proved both ideas that "unconscious simulation and conscious imagery are identical processes (e.g. Gallese \& Lakeoff, 2005)"[40] and that the vertification of action verbs associated with handedness upholds the egocentric possibility, indicating that when people comprehend action words, they tend to have an implicit simulation of their previous or potential actions (cited Willems, Hagoort, \& Casasanto, 2009). As a result, through these tests, the BSH gets more concrete evidence.

\subsection{Action and Emotion Valence}

When we were born as a baby, individuals try to choose things they expect to be positive and avoid negative things. This common cognition is a behavior which mankinds share with the most straightforward of organisms [15]. Meanwhile, from different cultures and conventions, people behave differently. For instance, you cannot shake hands with your left hand in Thailand because they think it is feculent. This phenomenon can use the BSH that in a sense, the content of our ideology is determined by the structure of individuals' bodies and within a predictable range, persons with various types of bodies ought to have different thoughts (cited Casasanto, \& Jasmin, 2010). However, through the test of Casasanto and Kyle Jasmin (2010) [15], the results show that "both culture and physical experience can potentially account for the mapping of "good is right" in gestures of right-handed people, whereas only physical factors can explain the "good is right" mapping found in gestures of left-handed people", indicating that action and emotion have a hidden relationship. Although culture or conventions cannot be explained, we can still know that emotion valence as an abstract concept of things seems to be in the basis of body-specific motor experience, which extends the BSH.

\subsection{Approach Motivation and Motor Control}

Define abbreviations and acronyms the first time they are used in the text, even after they have been defined in the abstract. Abbreviations such as IEEE, SI, MKS, CGS, sc, dc, and rms do not have to be defined. Do not use abbreviations in the title or heads unless they are unavoidable. With respect to previous studies on emotional motivation in the human brain, it is universally acknowledged that the prokinetic state was mainly supported by the left hemisphere, while the avoidance state was supported by the right hemisphere[6]. Based on the studies of Brookshire and Casasanto $(2012 ; 2018)[6][7]$, their papers give us evidences that specialization for approach motivation reverse with handedness and try to explain them on account of the Sword and Shield Hypothesis by using resting-state electroencephalography and transcranial direct current stimulation. The results reveal that the hemispheric correlation of approach motivation between left-handed and right-handed is opposite. This is because they usually use dominant and non-dominant hands to perform approach and avoidance actions in the same way [6]. Additionally, the tests indicate that proximity motivation is lateralized to the identical hemisphere which conducts the dominant hand in terms of both leftand right-handers[6], which supports the SSH. Moreover, what can we know is that "the large cortex of abstract concepts correspondingly organizes the way people interact with the world with their hands" [6]. These studies all give the evidence to uphold the $\mathrm{BSH}$, persons with different bodies have a different interaction with the environment in a systematic way, which can produce homologous differences in their thoughts and brains (cited Brookshire, \& Casasanto, 2018). 


\subsection{Visuospatial Attention}

In order to further extend the BSH associated with handedness, Colman, Remington and Kritikos (2017) endeavored to explain how factors referred to the internal representation of the hands involving handedness and grasping affordances affect the distribution of visuospatial attention near the body by using three experiments. Meanwhile, they tested "the additional contributions of handedness, hand gesture and proximity by systematically changing the relationship between action goal and hand proximity". Therefore, it figures out that "when completing a distal task, exogenous cues modulated attention" [18]. On the contrary, the laterality of the corresponding hand or handedness does not systematically affect attention in the first experiment. Second, the findings show that approaching the visual display, the grasping affordances of the right hand tends to shift in visuospatial attention. Besides, right-handers show faster participation and slower visual spatial attention away from the target near the hand, which was their dominant hand-grasping region. What is more, only the right-handers keep the dominant hand bias in terms of "accuracy of target identification and grasping space" [18] when hands cross the midline of the body in third experiment. Overall, these findings all provide the evidence to indicate that visuospatial attention not only has an intimacy link with the proximity between the objects and the hands, but also is correlative with grasp affordance and the strength of the underlying representation. More importantly, it also extends the BSH.

\subsection{Semantics: Metaphor Explanation}

Through testing whether gestures of left hands strengthen metaphor interpretation by Paraskevi, Sotaro and Christine (2017), which refers to the process of right hemisphere, they really gave some evidence from two experiments to support the BSH associated with handedness. On one hand, that individuals keep one specific hand still while they do spontaneous gestures by other hand shows that in the case of free left hand, the metaphor of spontaneous gestures in trails is higher than that of those without spontaneous gestures [4]. Unfortunately, they cannot find any link between the performance and gesturing in the condition of free right hand. Nevertheless, metaphorical phrases can be explained by right-handers, for instance, "to spill the beans" [4] and here "beans" symbolizes fragments of information. On the other hand, metaphor explanations were better when individuals use their right hand to do gestures than they do not use that at all. Similarly, there is no significant difference between right hand gesture condition and no-gesture condition. To be rigorous, they measured individual differences in determining the degree to which the right hemisphere involved in speech production with their mouth asymmetry. Then, it can be seen that on metaphor explanation, "the dominance of left-over-right-side mouth dominance is positively correlated with the dominance of left-over-right-hand gesture" [4]. Therefore, both of these two experiments supported the BSH associated with handedness.

\section{OTHER BODY ATTRIBUTES: LATERALITY, FLUENCY, PropriocePTION}

According to BSH's assumption, the semantic meaning of words is "constituted" by unique perceptions or movements differentiated among individuals. Therefore, individuals should have distinct neurocognitive representations of certain concepts since the perception and action they conduct in response to the external stimulus are systematically different due to the specific features of their own bodies [16]. While handedness has been considered the primary testbed, experimenting with the BSH so far, researchers have also tried to verify the theory targeting other body parts or attributes. The targets include but are not limited to footedness [20], arm fluency [26], and pain perception [31]. In these studies, participants showed neurocognitive differences (or conversions) during the process with similar tendencies regarding the common body characteristics they share, which potentially validates the BSH theory. Furthermore, some of these experiments were conducted while the experimenters were not necessarily aware of the BSH theory itself. Thus, sufficient results may have already supported the BSH theory without the experimenters' original intention, especially experiments conducted in physiological and medical domains.

\subsection{Footedness and Valence: Extending the Laterality Tested}

Considering the association between handedness and cerebral lateralization [24], the experiment researching relationship between footedness and valence is arguably the most relevant study directly targeting the BSH, which is primarily based on handedness, as both essentially focuses on the neurophysiological pathway relating body parts and the left and right hemisphere of the cortex. Specifically, the cerebral lateralization is activated distinctly for the right-handed and left-handed, validated in a silent word generation test displayed by fMRI [33]. Interestingly, though the bias towards right is slightly smaller (around 80\%) compared to handedness (around 90\%), there is evidence that footedness might be the optimal indicator of cerebral lateralization compared to other lateral preferences partially due to the social pressure factor [22]. Therefore, experiments have proposed the association between handedness and footedness, which further suggests that footedness, as 
one of the body specificities, affects people's semantic representation [16].

The plausible assumption demonstrating the handedness and valence is motor fluency or dominance [31], where the dominant hand is more associated with positive affect and vice versa. Compared to handedness, footedness does not show the influence as significant as handedness in human activities, but people are still hypothesized to show the difference in respect to the motor fluency between both of their feet, despite the difference pronounced minor. This difference should then deduce the correlation of dominant foot and positive valence. The experiment is conducted as the right-footer, and the left-footer made a valence judgment by using their foot to press the mechanical key on each side, which emerges a valence-by-side interaction [21]. As a result, in general, for positive phrases, responses were quicker when the participants pressed their dominant foot, and for negative phrases, responses were quicker for the nondominant foot.

Furthermore, the experimenter classifies the participants not only by their footedness but also the degree of their footedness (only for right-footers due to insufficient participants), and the result turns out that the interaction between the footedness and valence has only emerged for the strong group. The result supports BSH, as the individual's physical characteristics impact their cognitive representation. In addition, the strong-weak footedness classification also provides crucial support to the motor fluency assumption. More experiments are needed to corroborate the motor fluency assumption, which should literally address the relationship between the degree of lateralization and the valence judgment. An example would be the experiment targeting the association of the MARC effect and degree of handedness [25].

\subsection{Fluency and Creativity}

The two studies mentioned have covered the laterality of human bodies and built up the assumption of the role played by motor fluency in the BSH. However, their target mental representation is limited to the association of valence and laterality. In this study, the experimenter essentially focused on the relationship between motor fluency and divergence of creative thinking [29] from an embodied perspective. The idea is originated from the metaphorical theory, where abstract concepts are mapped onto superficially dissimilar concrete concepts. Therefore, it is hypothesized that the physical experience, such as arm movement of fluidity, would lead to more flowing, creative thought than nonfluidity. Specifically, participants performed fluid or non-fluid arm movements by tracing curved or angular line-drawings. They were then instructed to complete a creativity task generating alternative uses and mood, difficulty ratings, and demographic questions [36]. Generally, the results showed that participants in portraying curved line-drawings (i.e., fluid condition) subsequently came up with more alternative uses of printed journals than did those who traced angular linedrawings, involving in non-fluid condition. A clear distinction to be made here is that the new experiment focuses on the divergence [26] rather than both divergence and originality [36]. The result suggests that fluid movement influences cognitive processing [36].

Furthermore, some experiments have shown that the effect of fluid movements can be extended from divergent creative thinking to whole-body movements [26], where a bidirectional link between proprioceptivemotor kinematics and abstract metaphorical concepts exists. Though the experiments did not directly mention the BSH, their results align with the hypothesis, as the "fluidity" is a typical physical experience according to the environment. Even though it did not explicitly connect such physical experience with the specific body characteristics, the experiments certainly provide a direction of impairments on fluidity, especially based on the precedent examples in handedness and footedness. In other words, the impairments on fluidity were achieved through instructed, unnatural movements, while these impairments can also be implemented through intrinsic properties of human bodies, such as armlessness and leglessness [23]. The hypothesis can be reasonably deduced as the fluidity becomes severely limited because of bodily-specific abnormalities, the advanced cognitive process might be affected. A particular limitation to be noticed that the baseline comparison (non-movement) was not addressed in the original experiment [36] nor the replication [26], which may confuse the credibility of these experiments. Additionally, potential factors were not seriously controlled, gender [1] especially. Other limitation includes uncontrolled difference of participants in ethics and criteria of creativity rating [37].

\subsection{Pain Perception: Out of the Fluidity Box}

The significance of body specificity is first officially hypothesized in consideration of the relationship between bodily specific structure and emotional valence [16]. Studies mentioned have focused on the fluidity of movement associated with positive emotional feedback. In contrast, arguably, there were already studies researching the association between physical attributes and rather negative emotional valance. Though some of these studies did not show specific interest in BSH, their result suggests other evidence for the theory. Studies have shown that moral disgust elicited by unfair social circumstances shares similar facial muscular activities with more basic disgust related to contamination and toxin [17]. Such similarities suggest a link between higher-level cognitive skills (rejection against 
immorality) and physiological discomfort (vomiting toxin, visual stimulation of contamination), which is in line with the metaphorical theory. Secondly, experiments have researched the relationship between visual body appearance and pain perception. A negative body image, simulated by an injured rubber hand, shows effectiveness in increasing participants' pain sensitivity [31].

Additionally, some studies have shown that inhibiting furious statements may increase perceived pain afterward [10]. Therefore, advanced cognitive activities (i.e., moral judgment) shared their origin with the physiological reaction (i.e., discomfort), which is associated with specific bodily attributes (i.e., pain perception). Such deduction suggests a potential link between cognitive activities and physical attributes, supporting the BSH. However, the link remains subtle and purely, reversely theoretical because body specificity was not the clear focus of the experiments. Further examination on the direct linkage between the bodily characteristics and cognitive abilities is required, and experiments are expected to be conducted to make the bodily-specific difference (i.e., pain sensitivity) as the independent variable. In contrast, cognitive testing should be the dependent variable. To conclude, as BSH suggests, physical body characteristics influence neurocognitive representations; the pain perception experiment had inspired researchers to review other physical attributes, expanding supportive evidence for the BSH.

\section{Conclusion And Future Design}

Based on the theory and evidence we have mentioned above, BSH has obtained the evidence that physical features affect the representation of concepts. People with different physical characteristics have different minds. Even highly abstract thinking depends to some extent on the physical experience of the human body as a result of its interaction with the environment. At the same time, the effect of the body shaping the mind has stability and dynamic variability on a time scale. Of course, there are many factors influencing the process of body experience shaping cognition, and these factors will become the focus of research in the future. Here we will also provide some possible influencing factors and other research areas related to $\mathrm{BSH}$, including cross-cultural contexts, emotions, motivation and disability, in the hope of providing some inspiration for future researchers.

In summary, $\mathrm{BSH}$ is one of the important research fields of embodied cognition, and it is necessary and worthy of further discussion. BSH will not only be important evidence and application of embodied cognition, but also have realistic value. For example, in terms of publicity and advertising, valence is associated with spatial orientation to promote sales and improve visibility; In terms of social equity, order or location should not influence some elections that are only marginally different. In the future, more applied studies will be needed, and the study on the neural and behavioral internal mechanisms of BSH is not fully explored, so the research in this field will be of great value.

\subsection{Cross-cultural context}

Metaphors for abstract concepts vary across cultures. For example, individuals with various cultures, namely, from Spanish and Moroccan have different levels of taboo against using the left hand, and therefore have different preferences for the right hand. Similarly, plenty of languages and cultures link the right-hand side with wonderful events and the left-hand space with bad events. This pattern is reflected in linguistic statements such as "my right hand" and "two left feet", as well as in cultural practices that require people to eat or gesture with their right hand instead of their left [30]. Some researchers have discussed $\mathrm{BSH}$ from the perspective of culture. Unfortunately, there is no evidence of crosscultural differences between people of Spanish and Moroccan cultures in the implicit correlation between space and valence [19]. We think it's likely because the manipulation was not sensitive enough, because participants from both cultures still associated "good" with "right" despite the fact that there were varying degrees of handedness. Although the result is not significant, it is still a good revelation that we should pay attention to the regulation effect of culture and custom factors on BSH. We can find more valuable and conflicting metaphors. For example, in China, nodding is seen as affirmative or agreement, while in India, nodding is seen as negative or disagreement. At the same time, previous studies are only limited to the metaphorical connection between space and valence, and we can also explore other culture-specific metaphors for abstract concepts. The discussion of these cultural factors will contribute to a better understanding of $\mathrm{BSH}$ at evolutionary, social, and customary levels.

\subsection{Relationship of emotion and motivation}

On the whole, previous studies support the hypothesis that idiosyncratic hands affect emotional information coding and better account for the scope and nature of body-specific effects on spatial memory' [9]. However, our studies on spatial coding which refers to spatial location associated with handedness are still scarce. Perhaps, we can further extend visuospatial attention with emotion and motivation by other personal bodily experiences except for handedness to examine whether there is any internal link between them to give more evidence to support the BSH. 


\subsection{Impairments and Neuro Abnormalities}

As mentioned in the "Other Body Attributes, laterality, fluency and Proprioception" section, though plenty of experiments agreed with the theory, direct and decisive evidence is insufficient. Generally speaking, some of the previous studies, which did not directly refer to the BSH, were conducted chiefly focusing on the cognitive activities' influence on physiological attributes, while a direct demonstration requires a reversed order in experimenting. In other words, participants would be classified according to their distinctive physical characteristics first, and their advanced cognitive skills would be examined. Though human beings have different body attributes in general, significant distinguishments would be ideal for experimenting, including and not limited to amputation, congenital analgesia [33], color-blindness, and deafness [22]. Hypothetically, this physical distinctiveness would affect participants' neurocognitive representation pathway and make them "thinking differently" [11]. Interestingly, symptoms such as phantom (limb) pain have suggested a counterclaim towards the BSH: neurocognitive proprioception sustain to some extent regardless of the change in body operation (i.e., after being amputated) [28]. Indeed, the BSH has shown success explaining the relationship between physical body and cognition but taking the non-supportive circumstance into consideration would be essential to universalize the model.

\section{ACKNOWLEDGMENT}

We would like to acknowledge the valuable feedback and suggestions provided by the Daniel Casasanto, the Associate Professor of Human Development and Psychology at Cornell University, as well as a platform for us to have the opportunity to complete this work provided by Cathaypath Institute of Science.

\section{REFERENCES}

[1] Abraham, A., Thybusch, K., Pieritz, K. et al. (2014). Gender differences in creative thinking: behavioral and fMRI findings. Brain Imaging and Behavior, 8, 39-51.

[2] Andrew, K. N., Hoshooley, J., \& Joanisse, M. F. (2014). Sign Language Ability in Young Deaf Signers Predicts Comprehension of Written Sentences in English. PLoS One, 9(2).

[3] Aren, G., Sepet, E., Erbay, E., \& Ermis, I. (2002). Congenital analgia syndrome: Official Journal of the Society of the Nippon Dental University. Odontology, 90(1), 0064-8.
[4] Argyriou, P., Mohr, C., \& Kita, S. (2017). Hand matters: Left-hand gestures enhance metaphor explanation. Journal of Experimental Psychology: Learning, Memory, and Cognition, 43(6), 874-886. http://dx.doi.org/10.1037/xlm0000337

[5] Barsalou, L. (1999). Perceptual symbol systems. Behavioral and Brain Sciences, 22, 577-609.

[6] Brookshire, G., \& Casasanto, D. (2012). Motivation and motor control: Hemispheric specialization for approach motivation reverses with handedness. PLoS ONE, 7(4), 5. http://dx.doi.org/10.1371/journal.pone.0036036

[7] Brookshire G, \& Casasanto D. (2018). Approach motivation in human cerebral cortex. Phil. Trans. R. Soc. B 373: 20170141. http://dx.doi.org/10.1098/rstb.2017.0141

[8] Brouillet, D., Milhau, A., \& Brouillet, T. (2015). When "good" is not always right: Effect of the consequences of motor action on valence-space associations. Frontiers in Psychology, 6, 8. http://dx.doi.org/10.3389/fpsyg.2015.00237

[9] Brunyé, T. T., Gardony, A., Mahoney, C. R., \& Taylor, H. A. (2012). Body-specific representations of spatial location. Cognition, 123(2), 229-239. http://dx.doi.org/10.1016/j.cognition.2011.07.013

[10] Burns, J. W., Quartana, P. J., \& Bruehl, S. (2008). Anger inhibition and pain: Conceptualizations, evidence and new directions. Journal of Behavioral Medicine, 31(3), 259-79.

[11] Casasanto D. (2011). Different Bodies, Different Minds: The Body Specificity of Language and Thought. Current Directions in Psychological Science, 20(6), 378-383

[12] Casasanto, D., \& Chrysikou, E.G. (2011). When left is "right": Motor fluency shapes abstract concepts. Psychological Science, 22, 419-422.

[13] Casasanto, D., \& Lupyan, G. (2011). Ad hoc cognition. In L. Carlson, C. Hölscher, \& T. Shipley (Eds.), Proceedings of the 33rd Annual Conference of the Cognitive Science Society (p. 826). Austin, TX: Cognitive Science Society.

[14] Casasanto, D., \& Henetz, T. (2011). Handedness shapes children's abstract concepts. Cognitive Science [e-pub before print]. DOI: 10.1111/j.15516709.2011.01199

[15] Casasanto, D., \& Jasmin, K. (2010). Good and bad in the hands of politicians. PLoS ONE, 5(7), e11805. doi:10.1371/journal .pone.0011805 
[16] Casasanto, D. (2009). Embodiment of abstract concepts: Good and bad in right- and left-handers. Journal of Experimental Psychology: General, 138, $351-367$.

[17] Chapman, H. A., Kim, D. A., Susskind, J. M., \& Anderson, A. K. (2009). In Bad Taste: Evidence for the Oral Origins of Moral Disgust. Science, 323(5918), $1222-1226$. https://doi.org/10.1126/science.1165565

[18] Colman, H. A., Remington, R. W., \& Kritikos, A (2017). Handedness and graspability modify shifts of visuospatial attention to near-hand objects. PLoS ONE, 12(1), 19 http://dx.doi.org/10.1371/journal.pone.0170542

[19] de la Fuente, J., Casasanto, D., Román, A., \& Santiago, J. (2015). Can culture influence body-specific associations between space and valence? Cognitive Science, 39(4), 821-832. http://dx.doi.org/10.1111/cogs.12177

[20] de la Vega, I., Graebe, J., Härtner, L., Dudschig, C. \& Kaup, B. (2015). Starting off on the right foot: strong right-footers respond faster with the right foot to positive words and with the left foot to negative words. Frontiers in psychology, 6, 292.

[21] de la Vega, I., de Filippis, M., Lachmair, M., Dudschig, C., \& Kaup, B. (2012). Emotional valence and physical space: Limits of interaction. Journal of Experimental Psychology: Human Perception and Performance, 38(2), 375385. http://dx.doi.org/10.1037/a0024979

[22]Elisa L. J., Bryden M. P., Bulman-Fleming M. B. (1998). Footedness is a better predictor than is handedness of emotional lateralization. Neuropsychologia, 36, 37-43. http://dx.doi.org/10.1016/S0028-3932(97)00107-3

[23] Eshraghi, A., Noor Azuan, A. O., Karimi, M., Gholizadeh, H., Soodmand, E., \& Wan Abu Bakar, W. A. (2014). Gait Biomechanics of Individuals with Transtibial Amputation: Effect of Suspension System. PLoS One, 9(5).

[24] Hellige JB, Bloch MI, Cowin EL, Eng TL, Eviatar Z, \& Sergent V. (1994). Individual variation in hemispheric asymmetry: multitask study of effects related to handedness and sex. J Exp Psychol Gen. 123(3):235-56

[25] Huber S., Klein E., Graf M., Nuerk H. C., Moeller K., \& Willmes K. (2014). Embodied markedness of parity? Examining handedness effects on parity judgments. Psychol. Res. 1-15

[26] Imaizumi, S., Tagami, U., \& Yang, Y. (2020). Fluid movements enhance creative fluency: A replication of Slepian and Ambady (2012). PLoS One, 15(7).

[27] Kuo, Chun-Yu \& Yeh, Yei-Yu (2016). Sensorimotor-Conceptual Integration in Free Walking Enhances Divergent Thinking for Young and Older Adults. Frontiers in psychology, 7, 1580

[28] MacLachlan, M., Desmond, D., \& Horgan, O. (2003). Psychological correlates of illusory body experiences. Journal of Rehabilitation Research and Development, 40(1), 59-65.

[29] Malinin Laura H. (2019). How Radical Is Embodied Creativity? Implications of 4E Approaches for Creativity Research and Teaching. Frontiers in Psychology, 10, 2372

[30] McManus, I. C. (2002). Right hand, left hand: The origins of asymmetry in brains, bodies, atoms and cultures. London, UK / Cambridge, MA: Weidenfeld and Nicolson / Harvard University Press.

[31] Oppenheimer, D. M. (2008). Effects of Fluency on Psychological Distance and Mental Construal (or Why New York Is a Large City, but New York Is a Civilized Jungle). Psychological Science, 19(2), 161-167.

[32] Osumi, M., Imai, R., Ueta, K., Nobusako, S., \& Morioka, S. (2014). Negative body image associated with changes in the visual body appearance increases pain perception. PLoS One, 9(9)

[33] Parrott, L. M., C.R.N.A. (2013). Anesthetic Management of a Patient With Congenital Insensitivity to Pain: A Case Report. AANA Journal, 81(5), 376-8.

[34] Pujol J, Deus J, Losilla JM, Capdevila A. (1999). Cerebral lateralization of language in normal lefthanded people studied by functional MRI. Neurology, 52(5), 1038-43.

[35] Simon, H. A., \& Newell, A. (1964). Information processing in computers and man. American Scientist, 52, 281-300.

[36] Slepian, M. L., \& Ambady, N. (2012). Fluid movement and creativity. Journal of Experimental Psychology, 141(4), 625.

[37] Sternberg, Robert J. (1985). Implicit Theories of intelligence, creativity, and wisdom. Journal of Personality and Social Psychology, 49(3), 607-627.

[38] Tversky, B., \& Hard, B. M. (2009). Embodied and disembodied cognition: Spatial Perspective-taking. Cognition, 110, 124-129. 
[39] Willems, R.M., Labruna, L., D’Esposito, M., Ivry, R., \& Casasanto, D. (2011). A functional role for the motor system in language understanding: Evidence from theta-burst transcranial magnetic stimulation. Psychological Science, 22, 849-854.

[40] Willems, R. M., Hagoort, P., \& Casasanto, D. (2010). Body-specific representations of action verbs: Neural evidence from right- and lefthanders. Psychological Science, 21(1), 67-74. http://dx.doi.org/10.1177/0956797609354072

[41] Ye, H. (2010). Embodied Cognition: A New Orientation of Cognitive Psychology. Advances in Psychological Science, 18(5), 705-710.

[42]Zhao, X., He, X., Zhang, W., Chen, G., Chen, Q., \& Huang, L. (2018). Interpersonal choice: The advantage on the left or on the right? International Journal of Psychology, 53(5), 331-338. http://dx.doi.org/10.1002/ijop.12388 